\title{
SOME CONDITIONS ON FIXED RINGS
}

\author{
EDNA E. REITER \\ Department of Mathematics \\ University of Cincinnati \\ Cincinnati, Ohio 45221 \\ U.S.A. \\ (Received February 12, 1979)
}

ABSTRACT. This paper deals with a question about the ascending and descending chain conditions on two-sided ideals. Using the ideas of the skew group ring, certain results for two-sided ideals are proved.

KEY WORDS AND PHRASES. Fixed rings, ascending and descending chain conditions or two-sided ideals, and skew group ring.

\section{MATHEMATICS SUBJECT CLASSIFICATION CODES:}

1. INTRODUCTION.

Let $R$ be an associative ring with an identity element 1 , and let $G$ be a group of automorphisms acting on $R$ such that $|G|$, the order of $G$, is finite. In this paper we will prove several relationships between $R$ and the fixed ring $R^{G}=$ $\left\{r \in R: r^{g}=r\right.$ for all $\left.g \in G\right\}$. We answer the question raised by Fisher and Osterburg [7], that is, we prove that if $R$ has no $|G|$ - torsion (for $r \in G$, $|G| r=0$ implies $r=0$ ), and if Soc $R$ denotes the left (or right) socle of $R$, 
then we have (Soc $R$ ) $\cap R^{G} \subseteq$ Soc $R^{G}$. Thus for a semiprime ring with no $|G|-$ torsion, Soc $R / / R^{G}=\operatorname{Soc} R^{G}$.

We also answer a question about the ascending and descending chain conditions on two-sided ideals. It is well known that if $|G|^{-1} \in R$ (i.e., if $|G|$ is invertible in $R$ ) and if $R$ satisfies the ascending or descending chain condition on left (right) ideals, then $R^{G}$ also satisfies the same condition. However, the techniques used for one-sided ideals have not been applied to twosided ideals. In this paper, we prove the analogous result for two-sided ideals by using the skew group ring of $R$ and $G, R * G=\sum_{g \in G} R u_{g}$. Addition in $R * G$ is componentwise; multiplication is given by the relations $\left(r u_{g}\right)\left(s u_{h}\right)=\left(r s^{g^{-t}}\right) u_{g o h}$, extended linearly. The inverse question, posed as follows, remains open: If $R$ is semiprime and $|G|^{-1} \in R$, and if $R^{G}$ satisfies the ascending (descending) chain condition on two-sided ideals, then must $R$ satisfy the ascending (descending) chain condition on two-sided ideals? The analogous question is true for onesided ideals.

For a left and right Noetherian ring, the Artin radical has been defined as the sum of all Artinian left ideals of the ring. If $R$ is semiprime and $|G|^{-1}$ $\epsilon R$, then $A(R)$ is defined if and only if $A\left(R^{G}\right)$ is defined. In the final section of this paper, we prove that under these conditions, $A(R) \cap R^{G}=A\left(R^{G}\right)$. 2. THE SOCLES OF $R$ AND $R^{G}$.

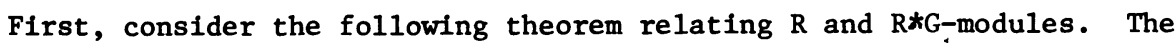
proof is a variation of the proof of Maschke's theorem. Note that all $R \star G-$ modules are certainly R-modules; on the other hand, the left $R^{*} G$-submodules of $R$ are the G-invariant left ideals of $R$ ( $I \quad \ell^{R}$ such that $I^{g} \leq I+g \in G$ ). THEOREM 2.1 Let $R$ be a ring and $G$ a finite group of automorphisms of $R$, and let $O \rightarrow N \rightarrow M \rightarrow N^{\prime} \rightarrow 0$ be a short exact sequence of left (right) $R^{*} G-$ modules such that the map $m \rightarrow|G| m$ is a bijection on $M$. Then if the sequence 
splits over $R$, it also splits over $R * G$.

PROOF. See Fisher-0sterburg [7, Theorem 1.3]. They assume $|G|^{-1} \in R$, but the proof can be carried out easily without this as long as for any $m \in M, n \in N$, there exist $m^{\prime}$ and $n^{\prime}$ in $M$ and $N$ respectively such that $|G|^{\prime}=m$ and $|G| n^{\prime}=n$.

From this, we obtain:

r.OROLLARY 2.2 Let $M$ be a left (or right) $R * G$-module with $|G|$ a bijection on M. If $M$ is semisimple as a left (or right) R-module, then $M$ is $R * G-s e m i s i m p l e$. PROOF. Immediate.

Now we can apply this to solve the socle question.

THEOREM 2.3 Let $R$ be a ring with no $|G|$-torsion, and let Soc $R$ denote the left (or right) socle of $R$. Then Soc $R \cap R^{G} \subseteq$ Soc $R^{G}$.

PROOF. We give the proof for the left socle only. Note that Soc $R$ is a Ginxariant left ideal of $R$; thus it is a left $R * G$-module. The simple components are minimal left ideals of $R$. These cannot be annihilated by $|G|$ so $|G|$ is a bifection on Soc $R$. We can apply Corollary 2.2: Soc $R$ is $R * G-s e m i s i m p l e, 1 . e .$, Soc $R=\sum_{i=1}^{n} \otimes K_{i}$, where each $K_{i}$ is a minimal G-invariant left ideal of $R$.

We now claim that each $K_{i} \cap R^{G}$ is a minimal left ideal of $R^{G}$. For, suppose that $X$ is a left ideal of $R^{G}$ such that $0 \neq x \subseteq K_{i} \cap R^{G}$. Then $0 \neq R X \subseteq R\left(K_{i} \cap\right.$ $\left.R^{G}\right) \subseteq K_{i}$; this implies $R X=K_{i}$. However, the fact that $|G| R X=R X$ forces $\mathrm{RX} \cap \mathrm{R}^{\mathrm{G}}=\mathrm{X}$, and the claim is proved.

Now, (soc $R) \cap R^{G}=\left(\sum_{i=1}^{n} \oplus k_{1}\right) \cap R^{G}$. Each $k_{1} \cap R^{G}$ is a minimal of $R^{G}$ and we will be finished if we can show that $\left(\sum_{i}^{n} \oplus K_{i}\right) \cap R^{G} \subseteq \sum_{i=1}^{n}\left(K_{i} \cap R^{G}\right)$. This is true, for suppose $\sum_{i=1}^{n} x_{i} \in R^{G}$, where $x_{i} \in K_{i}$. For any $g \in G, \sum_{i=1}^{n} x_{i}^{g}$ $=\sum_{i=1}^{n} x_{i}$. Thus, $x_{i}^{g}-x_{i}=\sum_{j \neq i} x_{j}^{g}-x_{j}=0, i=1, \ldots n$, since the sum is direct and each $k_{i}$ is $G$-invariant. These equations show that each $x_{i} \in R^{G}$.

REMARK. This theorem was subsequently proved by R. Diop [6], and, with the extra assumption that $|G|^{-1} \in R$, by M. Lorenz [9]. 
We note the following corollary.

COROLLARY 2.4 Let $R$ be a semiprime ring with no $|G|$-torsion. Then (Soc $R$ ) $\cap R^{G}=\operatorname{Soc} R^{G}$.

PROOF. By a theorem of Fisher and Osterburg [7], if $R$ is semiprime with no $|G|$-torsion, then Soc $R^{G} \subseteq$ Soc $R$.

\section{CHAIN CONDITIONS ON TWO-SIDED IDEALS.}

To deal with two-sided ideals in $R$ and $R^{G}$, we again consider the skew group ring. In particular, we will use the following lemma.

LEMMA 3.1 Let $R$ be any finite group. If $R$ has the ascending (descending) chain condition on two-sided ideals, then $R * G$ also satifies the ascending (descending) chain condition on two-sided ideals.

PROOF. The proof follows the method of the Hilbert Basis Theorem. For $G=\left\{g_{1}, \ldots g_{n}\right\}, I \quad R * G$, we define $I_{k}=\left\{r \in R\right.$ : for some $r_{1}, \ldots r_{k-1} \in R$, $\left.r_{1} u_{g_{i}}+\ldots+r_{k-1} u_{g_{k-1}}+r u_{g_{k}} \in I\right\}$ for $k=1, \ldots, n$. For a chain of ideals $I_{1}, I_{2}, \ldots I_{j}, \ldots$, let $I_{j, k}$ be the $k^{\text {th }}$ associated ideal of $I_{j}$ as defined above. The $n$ chains of ideals $I_{1, \ell}, I_{2, \ell}, \ldots I_{j, \ell}, \ldots, \ell=1, \ldots n$ must each terminate; this forces the chain $I_{j}$ in $R * G$ to terminate.

It may be mentioned that the above lemma is true for any ring $T$ and overring $s$ such that there exists a finite subset of $s,\left\{s_{1}, \ldots, s_{n}\right\}$ such that $S=\sum_{i=1}^{n} \mathrm{Ts}_{i}$ and $s_{i} \mathrm{~T}=\mathrm{Ts} \mathbf{i}_{i}$.

It is well known (and easily proved) that for any ring $S$ that has the ascending (descending) chain condition on two-sided ideals, any subring of the form eSe where $e^{2}=e$ must also satisfy the same condition. We use this fact, together with Lemma 3.1, to prove the following theorem.

THEOREM 3.2 If $|G|^{-1} \in R$ and $R$ satifies the ascending (descending) chain conditions on two-sided ideals, then $R^{G}$ satisfies the ascending (descending) chain condition on two-sided ideals. If $|G|^{-1} \in R$ and $R$ has a composition series of two-sided ideals, then $R^{G}$ has such a composition series also. 
PROOF. Consider the element $e=(1 /|G|) \sum_{G} u_{g} \in R * G$. It is easy to show

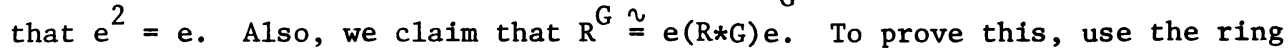
isomorphism $r \rightarrow\left(r u_{g_{1}}\right) e$, where $g_{1}$ is the identity of $G$ to obtain $R^{G} \cong R_{e}^{G}=e(R * G)$ e. Then, since $R * G$ has the ascending (descending) chain condition on two-sided ideals, so does $\mathrm{R}^{\mathrm{G}}$.

In the preceeding Theorem 3.2, we may weaken the assumption that $R$ has no $|G|^{-1} \in R$ to the assumption that $R$ has no $|G|$-torsion for the cases of the descending chain condition or the composition series property. This is true since $|G|$ is in the center of $R$ and $R|G|^{k}, k=1,2, \ldots$ is a descending chain of two-sided ideals which must terminate, forcing $|G|$ to be invertible in $R$. We state this as the following corollary.

COROLLARY 3.3. If a ring $R$ has no $|G|$-torsion, then if $R$ satisfies the descending chain condition (has a composition series of two-sided ideals), $R^{G}$ must also satisfy the descending chain condition (have a composition series of two-sided ideals).

PROOF. Evident from Theorem 3.2 and the paragraph above.

We conclude this section by noting an example due to Chuang and Lee [3]. This is a commutative Noetherian ring $R$ with involution, such that $R$ has no 2-torsion and the subring generated by the symmetric elements is not Noetherian. In a commutative ring, an involution is an automorphism of order 2 , and the subring generated by the symmetric elements is the fixed ring. This example, then, shows that for the ascending chain condition, the hypotheses of Theorem 3.2 cannot be weakened to assume merely no $|G|$-torsion.

4. THE ARTIN RADICALS OF $R$ AND $R^{G}$.

In a left and right Noetherian ring $R$, the Artin radical $A(R)$ is defined as the sum of all right ideals of $\mathrm{R}$ that are Artinian as right R-modules. This radical turns out to be a two-sided ideal of $R$; it is also equal to the sum of the left ideals of $R$ that are left R-Artinian. 
It is easy to prove that if $R$ is left and right Noetherian and if $|G|^{-1} \in R$, then $R^{G}$ is left and right Noetherian. Also, Farkas and Snider [5] have proved that if $R$ is semiprime with no $|G|$-torsion and if $R^{G}$ is left (right) Noetherian, then $R$ is left (right) Noetherian. Thus, for $R$ semiprime with $|G|^{-1} \in R, A(R)$ is defined if and only if $A\left(R^{G}\right)$ is defined. In this section we prove that under these conditions, $A(R) \Gamma\left(R^{G}=A\left(R^{G}\right)\right.$.

To prove the first inclusion, we need several preliminary facts. First, note that if $I$ is any G-invariant ideal of $R$, then $G$ (or a homorphic image of G) acts on $R / I$ by $g(r+I)=g(r)+I$. Furthermore, if $|G|^{-1} \in R$, it can be shown (see Fisher and Osterburg [7]) that $(R / I){ }^{G} \cong R^{G} /\left(I \cap R^{G}\right)$.

Also, we require an important theorem due to Bergman and Isaacs [1], which we state below. The trace of an element is defined by $\operatorname{tr} x=\sum_{g \in G} x^{g}$; the trace of an ideal $L$ is $\{\operatorname{tr} x: x \in L\}$.

THEOREM 4.1 (Bergman-Isaacs) Let $G$ be a finite group acting on a ring $R$ without $|G|$-torsion. Then:

(i) If $R$ is semiprime, then $R^{G}$ is semiprime.

(ii) If $R$ is semiprime and $L$ is a G-invariant left ideal of $R$ such that $t r$ $\mathrm{L}=0$, then $\mathrm{L}=0$.

Now we can prove:

THEOREM 4.2 If $R$ is semiprime and $|G|^{-1} \in R$, then $A(R) \cap R^{G} \subseteq A\left(R^{G}\right)$.

PROOF. First, we prove the following fact about right annihilators: $\left[r_{R}(A(R))\right]^{G}=r_{R}\left[A(R) \cap R^{G}\right]$. Only one inclusion requires proof.

Let $x \in \underset{r^{G}}{R}\left[A(R) \cap R^{G}\right]$; that is, $x \in R^{G}$ and $\left[A(R) \cap R^{G}\right] x=0$. Now $A(R) x$ is a G-invariant left ideal of $R$ and $\operatorname{tr}(A(R) x)=0$. The Bergman-Isaacs is applicable so we have $A(R) x=0$, i.e., $x \in\left[r_{R}(A(R))\right]^{G}$.

To prove the theorem, we show that $A(R) \cap R^{G}$ is an Artinian right ideal of $R^{G}$. By a theorem of Lenagan [8] if $I$ is an ideal of a left and right 
Noetherian ring such that $I$ is left R-Artinian, then $R / r_{R}$ (I) is an Artinian ring. Thus, $R / r_{R}(A(R))$ is an Artinian ring. Since $|G|^{-1} \in R$, it follows easily that the fixed ring of this factor ring is Artinian. Using this together with the facts on the preceeding page, we have the Artinian rings:

$$
\left[\frac{R}{r_{R}(A(R))}\right]^{G} \cong \frac{R^{G}}{r_{R}(A(R)) T / R^{G}}
$$

And, from the first part of the proof, we have $R^{G} /\left(r_{{ }^{G}}\left[A(R) \cap R^{G}\right]\right)$ is Artinian. It is true that a right ideal $I$ of a ring $S$ is contained in $A(S)$ if $S / r(I)$ is Artinian (see Chatters, Hajarnavis, and Norton [2], the proof of Theorem 1.3). Applying this, we have $A(R) \cap R^{G} \subseteq A\left(R^{G}\right)$.

To prove the other inclusion, we need the following theorem. Its proof is a variation of a proof by Cohen and Montgomery [4].

THEOREM 4.3 Let $R$ be a semiprime ring, left and right Noetherian with $|G|^{-1}$ $\in R$. If $A=A\left(R^{G}\right)$ is the Artinian radical of $R^{G}$, then $R A$ is an Artinian left R-module.

PROOF. Since $R$ is left and right Noetherian, there are left ideals of $R$, $K_{\alpha}$, maximal in $R A$. And, since ${ }_{\left(R^{G}\right)} A$ is artinian, we may choose a finite family of these, $k_{1}, \ldots, k_{n}$, to minimize $k=A \cap\left(k_{1} \cap \ldots \cap k_{n}\right)$.

We prove first that $K=0$. For, suppose not. Since ${ }^{A}$ is Artinian, the socle of $R^{G}$ is essential as a left ideal in A. Also, since $R^{G}$ is semiprime, Soc $\left(R^{G}\right)$ is a semisimple Artinian ring and has an identity element $1_{S}$. Now, if $\mathrm{K} \neq 0$ :

$$
K \cap \operatorname{Soc} R^{G}=\left(\operatorname{Soc} R^{G}\right) e \text {, where } e^{2}=e \neq 0
$$

Consider $R\left(1_{S}-e\right) \varsubsetneqq R A$. The inclusion is proper since the sum Re $R\left(1_{S}-e\right)$ $\subseteq \mathrm{RA}$ is direct, and equality would force $\mathrm{Re}=0$ and hence $\mathrm{K}=0$. Therefore, we can choose a left ideal of $R, K_{0}$, so that $K_{0}$ is maximal in $R A$ and:

$$
\mathrm{R}\left(1_{\mathrm{S}}-\mathrm{e}\right) \subseteq \mathrm{K}_{0} \varsubsetneqq \mathrm{RA}
$$


We now claim that $\mathrm{e} \mid \nexists \mathrm{K}_{0}$, which will make the ideal $\mathrm{R}^{\mathrm{G}} \cap\left(\mathrm{K}_{0} \cap \mathrm{K}_{1} \cap \ldots\right.$ $\left(\mid \mathrm{K}_{\mathrm{n}}\right)$ properly smaller that $\mathrm{K}$, contradicting the choice of $\mathrm{K}_{1}, \ldots, \mathrm{K}_{\mathrm{n}}$ and thus forcing $\mathrm{K}=0$. To prove the claim, suppose that $\mathrm{e} \in \mathrm{K}_{0}$. Then obviously $1_{S} \in \mathrm{K}_{0}$. Note that the sum $A=A 1_{S} \otimes A\left(1-1_{S}\right)$ is direct, and that Soc $R^{G}=$ $R^{G} \cdot 1_{S}$, so that Soc $R^{G} \cap A\left(1-1_{S}\right)=0$. Since Soc $R^{G}$ is essential in $A$, we obtain $\mathrm{A}=\mathrm{Al}_{\mathrm{S}}$. Thus, $\mathrm{RA}=\mathrm{R} 1_{\mathrm{S}} \subseteq \mathrm{K}_{0}$, and $\mathrm{RA}=\mathrm{K}_{0}$, a contradiction to the choice of $\mathrm{K}_{0}$.

Now consider $\bigcap_{i=1}^{n} \bigcap_{g \in G} K_{i}^{g}=L \subseteq R A$. Certainly L.is a G-invariant left ideal of $R$ and $L \stackrel{i=1}{\cap} \mathrm{g} \in \mathrm{G} \subseteq \mathrm{K}=0$. Also, $\mathrm{L} \Gamma \mathrm{R}^{\mathrm{G}} \subseteq \mathrm{RA} \cap \mathrm{R}^{\mathrm{G}}=\mathrm{A}$. We now have:

$$
L \cap R^{G} \subseteq L \cap A r_{R}^{G}=0
$$

Since $L \cap R^{G}=0$, certainly $\operatorname{tr}\left(L \cap R^{G}\right)=0$. By the Bergman-Isaacs theorem, $\mathrm{L}=0$. Thus, there is a natural $\mathrm{R}$-module embedding of $\mathrm{RA}$ in the finite direct sum of simple left R-modules, $\sum_{i=1}^{n} \sum_{g \in G} R A /\left(K_{i}^{g}\right)$. Now it is immediate that $R A$ has finite length as a left R-module.

We close with two corollaries.

COROLLARY 4.4 Let $R$ be left and right Noetherian, semiprime with $|G|^{-1}$

$\in R$. Then $A\left(R^{G}\right) \subseteq A(R)$.

PROOF. We have proved that $R A\left(R^{G}\right)$ is left Artinian; $A(R)$ is the sum of all Artinian left ideals. Thus, $A\left(R^{G}\right) \subseteq R A\left(R^{G}\right) \subseteq A(R)$.

COROLLARY 4.5 Iet $R$ be left and right Noetherian, semiprime with $|G|^{-1}: \in R$. Then $A\left(R^{G}\right)=A(R) \cap R^{G}$.

PROOF. Theorem 4.2 and Corollary 4.5. 


\section{REFERENCES}

1. G.M. Bergman and I.M. Isaacs, Rings with fixed-point free group actions, Proc. London Math. Soc., 27 (1973), pp. 69-87.

2. A.W. Chatters, C.R. Hajarnavis, and N.C. Norton, The Artinian Radical of a Noetherian Ring, J. Austral Math. Soc., 23 (Series A), (1977), pp. 379-384.

3. C.L. Chuang and P.H. Lee, Noetherian Rings with Involution, Chinese J. Math., $\underline{5}$ (1977), no. 1, pp. 15-19.

4. Miriam Cohen and Susan Montgomery, Semi-simple Artinian Rings of Fixed Points, Canad. Math. Bu11., 18(2) (1975), pp. 189-190.

5. Daniel R. Farkas and Robert L. Snider, Noetherian Fixed Rings, Pacific J. Math., 69 (1977), pp. 347-353.

6. Raoul Diop, Actions de Groupes, Doctoral Thesis, L'Universite de Poitiers, 1978.

7. Joe W. Fisher and James Osterburg, Some Results on Rings with Finite Group Actions, Ring Theory: Proceedings of the Ohie Univ. Conf, Marcel Dekker, Inc. (1977), pp. 95-111.

8. T.H. Lenagan, Artinian Ideals in Noetherian Rings, Proc. Amer. Math. Soc., 51 (1975), pp. 499-500.

9. Martin Lorenz, Primitive Ideals in Crossed Products and Rings with Finite Group Actions, Math. Z., to appear. 


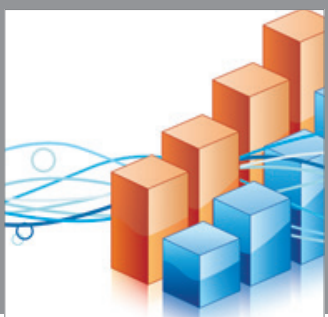

Advances in

Operations Research

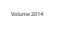

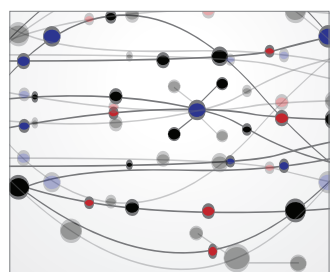

\section{The Scientific} World Journal
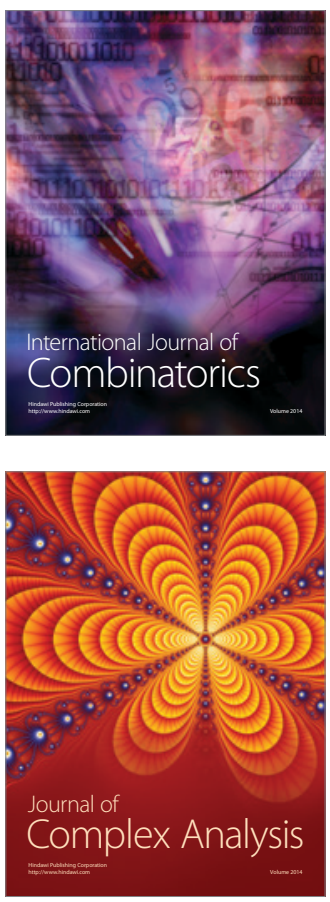

International Journal of

Mathematics and

Mathematical

Sciences
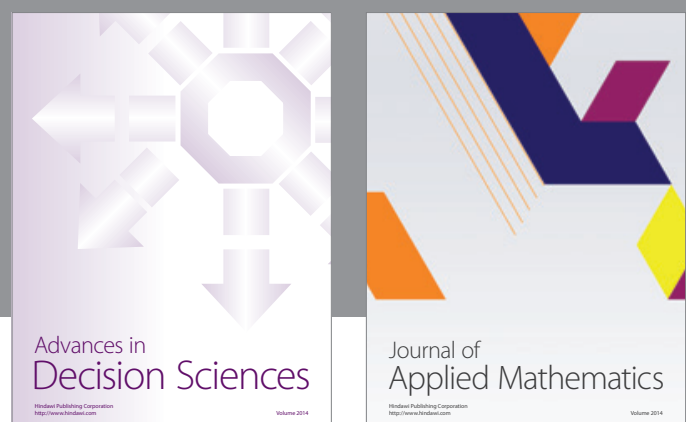

Journal of

Applied Mathematics
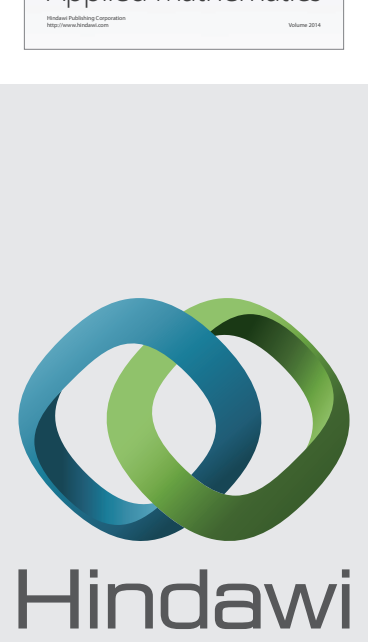

Submit your manuscripts at http://www.hindawi.com
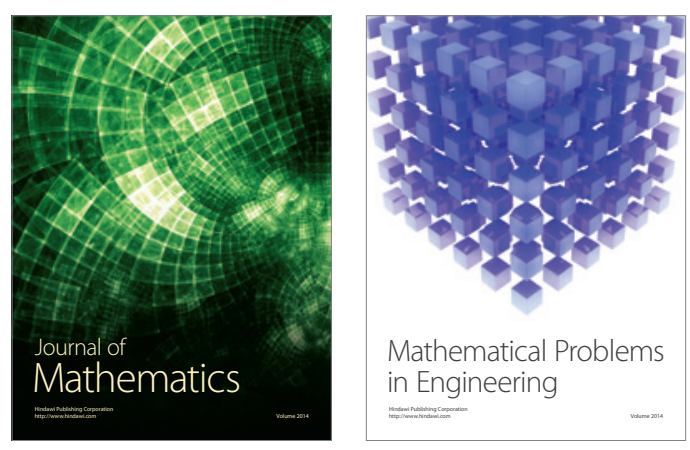

Mathematical Problems in Engineering
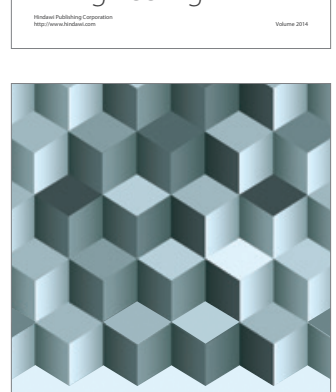

Journal of

Function Spaces
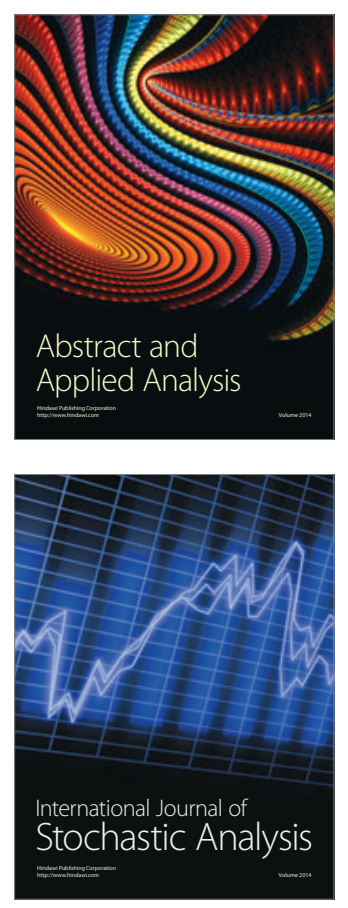

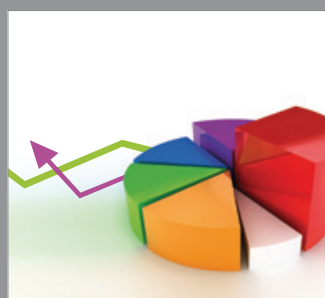

ournal of

Probability and Statistics

Promensencen
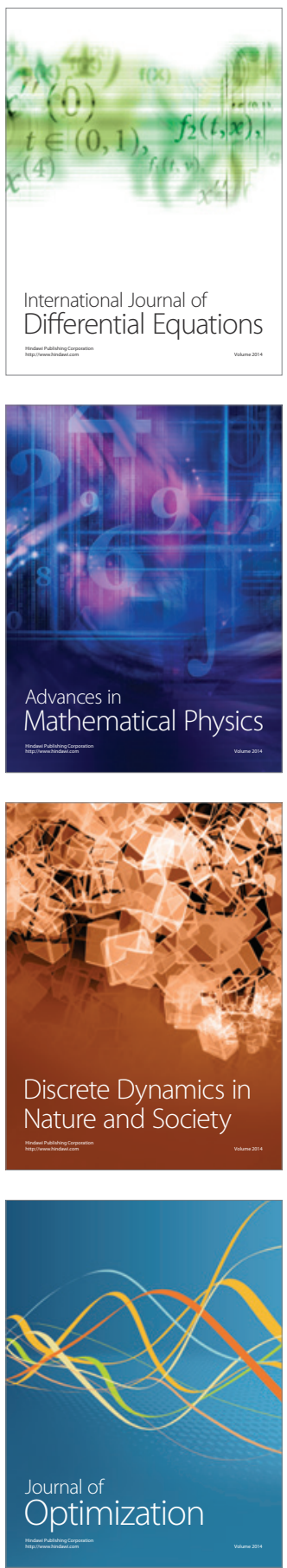